\title{
A commentary on comparison of the performance of lon Torrent chips in noninvasive prenatal trisomy detection
}

\author{
Osamu Samura \\ Journal of Human Genetics (2014) 59, 421-422; doi:10.1038/jhg.2014.56; published online 3 July 2014
}

$\mathrm{T}_{\mathrm{ar}}^{\mathrm{h}}$ he possibility of fetal chromosomal aneuploidy is a major reason why couples consider prenatal diagnostic studies. Conventional methods for definitive prenatal diagnosis of these disorders involve the invasive sampling of fetal materials through amniocentesis and chorionic villus sampling. However, these procedures involve some degree of risk for miscarriage and other pregnancy complications. ${ }^{1}$

Methods for noninvasive screening for fetal aneuploidy using maternal serum markers and ultrasound are available but have limited reliability. ${ }^{2}$ Therefore, it is desirable to develop noninvasive genetic tests for fetal chromosomal abnormalities. Intact fetal cells can be identified in maternal blood but are not a reliable source of fetal genetic material because they are extremely rare $^{3}$ and may persist for years after prior pregnancies. ${ }^{4}$ The ability to isolate fetal DNA from the plasma of pregnant women has opened up exciting opportunities for improved noninvasive prenatal testing (NIPT). ${ }^{5}$

Recent advances in DNA sequencing technology, as well as counting statistics, have provided an opportunity to develop new methods for the noninvasive detection of fetal aneuploidy. In 2008, Fan et al. ${ }^{6}$ described a quantification method for the noninvasive detection of fetal aneuploidy that involved counting chromosomes by mapping sequence tags generated through massively parallel sequencing of cell-free DNA (cfDNA) in maternal plasma, even without separating maternal and fetal cfDNA or enriching for

Dr O Samura is at Department of Obstetrics and Gynecology, The Jikei University School of Medicine, Tokyo, Japan

E-mail: osamusamura@gmail.com fetal cfDNA. Using massively parallel sequencing, millions of both fetal and maternal DNA fragments can be sequenced simultaneously, and given that the entire human genome sequence is known, each piece that maps to a discrete locus can be assigned to the chromosome from which it originated. If fetal aneuploidy is present, a relative excess or deficit should be observed for the chromosome in question. The number of fetal sequence reads will also vary according to the fetal fraction so that a minimum fetal fraction, typically $4 \%$, is currently required. Most studies have used Illumina sequence analyzers, but studies have also used SOLiD and Helicos platforms. Wang et al. ${ }^{7}$ reported that semiconductors enabling high-throughput sequencing, represented by Ion Torrent PGMTM/ProtonTM, proved to be feasible for noninvasive prenatal diagnosis of fetal aneuploidies. This approach has the advantage of requiring less sequencing and reduces costs.

Recently, other methods for NIPT have become commercially available. Targeted massively parallel sequencing enables the selective amplification of only the chromosomal regions of interest (for example, chromosomes 21, 18 and 13) and then the assessment of whether departure from euploidy is observed based on the relative number of DNA fragment counts for this subset of chromosomes. The major commercial device using this approach includes an adjustment that enables variation in the proportion of fetal DNA and then combines the results of the laboratory test with maternal age to provide patientspecific risk for Down, Edwards and Patau syndromes. ${ }^{8}$
The single-nucleotide polymorphism (SNP)-based approach involves the multiplex amplification of 11000 SNP sequences in a single PCR applied to plasma DNA, followed by sequencing. ${ }^{9}$ Each product is evaluated based on the hypothesis that the fetus is monosomic, disomic or trisomic. After consideration of the positions of SNPs on the chromosomes and the possibility of recombination, maximum likelihood is calculated to determine whether the fetus is either normal, aneuploid (chromosomes 21, 18 , or 13 or a sex chromosome) or triploid, or that uniparental disomy is present.

Noninvasive screening for aneuploidy using cfDNA in maternal blood was made available for clinical use in 2011. Worldwide, over 300000 NIPTs have been performed to date. Testing has shown $>99 \%$ sensitivity for the detection of trisomy 21 . When aneuploidy is detected, it is recommended that a follow-up invasive diagnostic procedure should be performed to determine the fetal karyotype. False-negative or inadequate sample results can occur when fetal cfDNA is at a too low concentration in maternal plasma if the assay fails to quantify the relative concentration of fetal cfDNA. False-positive results can be due to factors such as maternal aneuploidy, maternal cancer, vanishing twins, confined placental mosaicism or failure of the necessary complex bioinformatics to generate a result. Because most fetal cfDNA in maternal circulation is of placental origin, a discrepancy in the karyotype of the placenta and the fetus will lead to a discordant result. This phenomenon, confined placental mosaicism, is well known in prenatal diagnosis when results of chorionic villus sampling do not reflect the fetal 
karyotype and is an additional source of both false-positive and false-negative results for cfDNA.

Cell-free DNA has great potential for use in prenatal diagnosis. Noninvasive sequencing of the entire genome will be possible in the near future. ${ }^{10}$ The use of this technology should thus be carefully considered in clinical situations.

1 Mujezinovic, F. \& Alfirevic, Z. Procedure-related complications of amniocentesis and chorionic villous sampling: a systematic review. Obstet. Gynecol. 110 , 687-694 (2007).
2 Benn, P., Cuckle, H. \& Pergament, E. Non-invasive prenatal testing for aneuploidy: current status and future prospects. Ultrasound Obstet. Gynecol. 42, 15-33 (2013).

3 Bianchi, D. W., Williams, J. M., Sullivan, L. M., Hanson, F. W., Klinger, K. W. \& Shuber, A. P. PCR quantitation of fetal cells in maternal blood in normal and aneuploid pregnancies. Am. J. Hum. Genet. 61, 822-829 (1997).

4 Bianchi, D. W., Zickwolf, G. K., Weil, G. J., Sylvester, S. \& DeMaria, M. A. Male fetal progenitor cells persist in maternal blood for as long as 27 years postpartum. Proc. Natl Acad. Sci. USA 93, 705-708 (1996).

5 Lo, Y. M., Corbetta, N., Chamberlain, P. F., Rai, V., Sargent, I. L., Redman, C. W. et al. Presence of fetal DNA in maternal plasma and serum. Lancet 350 , 485-487 (1997)

6 Fan, H. C., Blumenfeld, Y. J., Chitkara, U., Hudgins, L. \& Quake, S. R. Noninvasive diagnosis of fetal aneuploidy by shotgun sequencing DNA from maternal blood. Proc. Natl Acad. Sci. USA 105 16266-16271 (2008)

7 Wang, Y., Wen, Z., Shen, J., Cheng, W., Li, J., Qin, X. et al. Comparison of the performance of Ion Torrent chips in noninvasive prenatal trisomy detection. J. Hum. Genet. 59, 339-396 (2014).

8 Sparks, A. B., Wang, E. T., Struble, C. A., Barrett, W., Stokowski, R., McBride, C. et al. Selective analysis of cellfree DNA in maternal blood for evaluation of fetal trisomy. Prenat. Diagn. 32, 3-9 (2012).

9 Zimmermann, B., Hill, M., Gemelos, G., Demko, Z., Banjevic, M., Baner, J. et al. Noninvasive prenatal aneuploidy testing of chromosomes $13,18,21, \mathrm{X}$, and $Y$, using targeted sequencing of polymorphic loci. Prenat. Diagn. 32, 1233-1241 (2012).

10 Fan, H. C., Gu, W., Wang, J., Blumenfeld, Y. J., El-Sayed, Y. Y. \& Quake, S. R. Non-invasive prenatal measurement of the fetal genome. Nature 487 320-324 (2012). 